\title{
Association of MLHI single nucleotide polymorphisms with clinical outcomes of first-line irinotecan-based chemotherapy in colorectal cancer
}

This article was published in the following Dove Press journal:

OncoTargets and Therapy

\author{
Deqiang Wang ${ }^{1, *}$ \\ Xiaomei Zhang ${ }^{2, *}$ \\ Yan Zhang ${ }^{2}$ \\ Yuan $\mathrm{Wu}^{2}$ \\ Xin Guan ${ }^{2}$ \\ Wei Zhu ${ }^{3}$ \\ Mei Wang ${ }^{3}$ \\ Chuang $\mathrm{Qi}^{4}$ \\ Bo Shen ${ }^{2}$
}

'The Cancer Therapy Center, Affiliated Hospital of Jiangsu University,

Zhenjiang, China; ' Jiangsu Cancer Hospital, Jiangsu Institute of Cancer Research, Nanjing Medical University Affiliated Cancer Hospital, Nanjing, China; ${ }^{3}$ School of Medicine, Jiangsu University, Zhenjiang, China; ${ }^{4}$ The Medical Department, 3D Medicines Inc., Shanghai, China

*These authors contributed equally to this work

\begin{abstract}
Purpose: Several studies have proved that single nucleotide polymorphisms (SNPs) of mismatch repair system genes are closely related to the development of colorectal cancer (CRC) by causing microsatellite instability, while effects of the SNPs of MMR system-related genes on the clinical outcomes of cytotoxic chemotherapy are less understood. The aim of this study explored the influence of MLH1 SNPs on clinical outcomes of first-line irinotecan-based chemotherapy in CRC.
\end{abstract}

Patients and methods: A total of 125 metastatic colorectal cancer (mCRC) patients who received first-line irinotecan-based chemotherapy (none of them combined with bevacizumab or cetuximab) were enrolled in this study. Blood samples or formalin-fixed paraffin-embedded tissues of study population were taken. DNA isolation and genotyping analyzed were obtained for potential functional polymorphisms of MLH1 rs1800734 by real-time PCR. Progressionfree survival (PFS) was the primary endpoint and tumor response rate (RR) was the secondary endpoint of this study.

Results: Of all the assessable population, the result showed no statistical difference among the three types SNPs of MLH1 rs 1800734 (AA, AG, GG) for RR ( $P=0.859)$, and also without significant difference for $\mathrm{AA}+\mathrm{AG}$ combined variants vs $\mathrm{GG}$ variant $(P=0.849)$. The median PFS for AA, AG, and GG variants of MLH1 rs1800734 SNPs were 9.4 months, 7.0 months, and 6.9 months, respectively (log-rank $P=0.031$ ). Interestingly, compared with AA variant of MLH1 rs1800734 SNPs, GG variant showed a shorter PFS (HR: 3.49; 95 CI: 1.02-11.94; $P=0.046$ ). Furthermore, the median PFS of AA $+\mathrm{AG}$ combined variants and $\mathrm{GG}$ variant were 8.3 months and 6.9 months (log-rank $P=0.037$ ), and $\mathrm{GG}$ variant have a decreased trend with no significant difference (HR: 1.57; 95 CI: $0.98-2.53 ; P=0.061$ ).

Conclusion: The AA variant of MLH1 rs1800734 SNPs has a longer PFS in first-line irinotecanbased chemotherapy for mCRC patients, and the result needs to be further confirmed by prospective studies in the future.

Keywords: MLH1 SNPs, irinotecan, colorectal cancer

\section{Introduction}

Department of Oncology, Jiangsu Cancer Hospital, Jiangsu Institute of Cancer Research, Nanjing Medical University Affiliated Cancer Hospital, No.42 Baiziting, Xuanwu District, 210009 Nanjing, Jiangsu, China

Tel +862583283554

Email shenboljs@I26.com
Colorectal cancer (CRC) is one of the most common gastrointestinal malignancies. According to the data from the National Cancer Center of China, 376,300 new cases of CRC were detected with 191,000 deaths in 2015, and with the number of new cases and deaths, it ranked fifth among the other types of human cancers. ${ }^{1}$ Even though chemotherapy or chemotherapy combined with targeted therapy bring clinical benefits, 
the treatment of CRC is still facing a severe situation. Previous Phase III studies showed irinotecan plus 5-fluorouracil/ leucovorin (5-FU/LV) was well tolerated and increased the response rate (RR), prolonged the overall survival (OS), and progression-free survival (PFS) compared with 5-FU/ LV-only treatment; but on the other hand, the RR for this combination therapy was only $35 \%-40 \%$, therefore, large proportion of patients could not benefit from this clinical therapy. ${ }^{2-4}$ Many studies have focused on the toxic effects of irinotecan-based chemotherapy, whereas few have also explored the benefits in the treatment of CRC. ${ }^{5-7}$

The mismatch repair (MMR) genes, mainly MLH1, MSH2, MSH6, and PMS2, can identify and repair errors during DNA replication and maintain chromosome stability. ${ }^{8}$ The status of the MMR system (evaluation by protein immunohistochemistry) included deficient mismatch repair (dMMR) system or proficient mismatch repair (pMMR) system, which played an important predictive factor in clinic treatment for metastatic colorectal cancer (mCRC) patients. For instance, stage II CRC patients with dMMR could not benefit from adjuvant 5-FU chemotherapy after surgery. ${ }^{9}$ Similarly, only dMMR mCRC patients could benefit from the treatment of immune checkpoint inhibitors. ${ }^{10,11}$

Interestingly, some researches also studied the relationship between the clinical efficacy of irinotecan-based chemotherapy and the state of MMR system in metastatic colon cancer, but the conclusion of these studies are controversial. ${ }^{12,13}$ Recently, the single nucleotide polymorphisms (SNPs) of MMR-related genes aroused our great interest. Several studies found that these polymorphisms may alter the function of DNA MMR system. ${ }^{14,15}$ For example, one of MLH1 SNP sites (rs1800734, MLH1-93G>A) is associated with promoter $\mathrm{CpG}$ island hypermethylation and microsatellite instability, contributing to CRC development. So far no researches reported the association of MLH1 rs1800734 SNPs with clinical outcomes of first-line irinotecan-based chemotherapy. In this study, we want to explore whether polymorphic locus of MLH1 rs1800734 can influence the clinical outcomes of first-line irinotecanbased chemotherapy in CRC.

\section{Patients and methods Study population}

From January 2016 to May 2018, 125 mCRC patients who received standard first-line irinotecan-based chemotherapy (none of them combined with bevacizumab or cetuximab) at Jiangsu Cancer Hospital were enrolled in the study. The included patients must meet all the following criteria: 1) written informed consent; 2) pathologically confirmed colorectal adenocarcinoma with complete clinical outcomes information; and 3) available blood samples or formalin-fixed paraffin-embedded (FFPE) tissues for PCRbased sequencing.

\section{Selected polymorphisms and genotyping}

Based on the previous published studies, the SNPs of MLH1 rs1800734 were selected for gene test and genotyping was performed using Taqman allelic discrimination assays. Genomic DNA was extracted from FFPE tissues or blood specimens from patients enrolled in this study. ${ }^{16}$ Primers used were F, 5'-ACCCAGCAACCCACAGAGT-3' and R, 5'-GTCTAGATGCTCAACGGAAGTG-3'. Probes used were C: FAM-TCTTCCTTCAGCTGTAG-MGB and T: HEXTTCTTCCTTTAGCTGTAGC-MGB. The investigator who read the sequence was blinded to the clinical outcome data.

\section{Statistical analyses}

Tumor responses were sorted according to the response evaluation criteria in solid tumors guidelines. The percentage of the patients obtaining complete response and partial response was considered as the RR. Patients who obtained stable disease and progressive disease are considered to be nonresponders. PFS was defined as the time from the first day of starting therapy to the first day of documented disease progression or death. The association between different SNPs and RR was assessed by Pearson's chi-squared test or Fisher's exact test. Log-rank test for PFS in the univariate analysis and Cox proportional hazards regression model in multivariate analysis were used. For all analyses, $P$-values were two-tailed and its value $<0.05$ was considered statistically significant.

\section{Statement of ethics}

Written informed consent was obtained from all the patients included in this study. The ethical committee of the Jiangsu Cancer Hospital approved the study, and all experimental procedures were performed in accordance with the 1964 Declaration of Helsinki and its later amendments or comparable ethical standards.

\section{Results}

\section{Patient characteristics}

The baseline characteristics of patients enrolled in this study are summarized in Table 1 . Out of the 125 patients included, $71(56.8 \%)$ were male and $54(43.2 \%)$ were female. 
Table I Patient demographics and clinical characteristics

\begin{tabular}{l|l}
\hline Patient characteristics & All patients $(\mathbf{n}=\mathbf{I} \mathbf{2 5})$ \\
\hline Gender & $71(56.8 \%)$ \\
$\quad$ Male & $54(43.2 \%)$ \\
Female & $59.14 \pm \mathrm{II.87}$ \\
Median age (years) & \\
ECOG PS & $67(53.6 \%)$ \\
0 & $58(46.4 \%)$ \\
I-2 & $76(60.8 \%)$ \\
Primary site & $49(39.2 \%)$ \\
Left colon or rectum & \\
Right colon & $91(72.8 \%)$ \\
Liver-limited metastases & $34(27.2 \%)$ \\
Yes & \\
No & $36(28.8 \%)$ \\
RAS status & $89(71.2 \%)$ \\
KRAS or NRAS mutant & \\
Wild-type & $70(56.0 \%)$ \\
Disease status & $55(44.0 \%)$ \\
Primary metastatic & \\
Recurrent & $125(100 \%)$ \\
Regimen &
\end{tabular}

Note: FOLFIRI is the combination of irinotecan with 5-FU/leucovorin.

Abbreviations: ECOG, Eastern Cooperative Oncology Group; PS, performance status.

The median age of the patients was $59.14 \pm 11.87$ years. The patient number of Eastern Cooperative Oncology Group performance status $0-1$ and $1-2$ were $67(53.6 \%)$ and $58(46.4 \%)$, respectively. Seventy $(56.0 \%)$ patients showed primary metastatic disease status at diagnosis and others showed recurrent disease status. Ninety-one (72.8\%) patients had a liver metastases. All of study population received RAS (KRAS and NRAS) gene testing in hospital, and KRAS or NRAS gene mutation occurred in $36(28.8 \%)$ patients. Different variants for MLH1 rs1800734 SNPs are listed in Table 2.The number of AA, GG, and AG variant of MLH1 rs1800734 SNPs was 7 (5.6\%), 77 (61.6\%), and 41 (32.8\%), respectively, in all the patients.

\section{Association of SNPs with clinical outcomes}

Overall response rate (ORR) and PFS were evaluated to find their relationship to different variants of MLH1 rs1800734 SNPs. The results of this part are summarized in Table 2.

\section{ORR}

The ORR was achieved in $3(42.8 \%)$ patients in AA variant, 14 (34.1\%) patients in AG variant, and 26 (33.8\%) patients in GG variant of MLH1 rs1800734 SNPs, with no significant difference among three groups $(P=0.859)$. The ORR of AA + AG combined variants and GG variant of MLH1 rs1800734 SNPs also did not show any significant difference ( $35.4 \%$ vs $33.7 \% ; P=0.849$ ), indicating no relationship between different alleles of MLH1 rs1800734 SNPs with tumor response in first-line irinotecan-based chemotherapy.

\section{PFS}

The median PFS for AA, AG, and GG variant of MLH1 rs 1800734 SNPs were 9.4 months, 7.0 months, and 6.9 months, respectively (log-rank $P=0.031$, Figure 1 ). Compared with AA variant, GG variant shows a shorter PFS in both univariate (HR: 3.73 ; $95 \mathrm{CI}: 1.15-12.15 ; P=0.028$ ) and multivariate analysis (HR: 3.49 ; 95 CI: $1.02-11.94 ; P=0.046$, Figure 2), whereas no statistically significant difference were found for $A G$ variants in both univariate (HR: 1.74; 95 CI: $0.95-3.22 ; P=0.075)$ and multivariate analysis (HR: 1.70; 95 CI: $0.91-3.20 ; P=0.098)$. These results indicate that AA variant of MLH1 rs1800734 SNPs has a better PFS in first-line irinotecan-based chemotherapy.

Table 2 Association between different genotypes for MLHI rs 1800734 and clinical outcomes in mCRC patients

\begin{tabular}{|c|c|c|c|c|c|c|c|c|c|}
\hline \multirow[t]{2}{*}{ SNP type } & \multirow[t]{2}{*}{$\mathbf{N}(\%)$} & \multicolumn{3}{|c|}{ Tumor response } & \multicolumn{5}{|l|}{ PFS } \\
\hline & & $\mathbf{P R}+\mathbf{C R}$ & SD + PD & $P$-value & $\begin{array}{l}\text { Median months } \\
(95 \% \mathrm{Cl})\end{array}$ & $P$-value & HR (95\% CI)* & HR $(95 \% \mathrm{Cl})^{\#}$ & $P$-value \\
\hline MLHI & & & & 0.859 & & 0.031 & & & \\
\hline $\mathrm{AA}$ & 7 (5.6\%) & $3(42.8 \%)$ & $4(57.2 \%)$ & & $9.4(9.1-9.7)$ & & I (Reference) & I (Reference) & \\
\hline AG & $4 \mathrm{I}(32.8 \%)$ & $14(34.1 \%)$ & 27 (65.9\%) & & $7.0(5.0-8.6)$ & & $1.74(0.95-3.22)$ & $1.70(0.91-3.20)$ & 0.098 \\
\hline GG & $77(61.6 \%)$ & 26 (33.8\%) & $5 \mathrm{I}(66.2 \%)$ & & $6.9(5.2-8.6)$ & & $3.73(1.15-12.15)$ & $3.49(1.02-11.94)$ & 0.046 \\
\hline & & & & 0.849 & & 0.037 & & & \\
\hline$A A+A G$ & $48(38.4 \%)$ & 17 (35.4\%) & 31 (64.6\%) & & $8.3(6.2-10.4)$ & & I (Reference) & I (Reference) & \\
\hline GG & $77(61.6 \%)$ & $26(33.7 \%)$ & $5 \mathrm{I}(66.3 \%)$ & & $6.9(5.2-8.6)$ & & $1.61(1.02-2.55)$ & $1.57(0.98-2.53)$ & $0.06 \mathrm{I}$ \\
\hline
\end{tabular}

Notes: $P$-value was based on chi-squared test or Fisher's exact test for tumor response. *Log-rank test for PFS and OS in the univariate analysis, and \#Wald test in the multivariate Cox proportional hazards regression model adjusting for sex, age, ECOG PS, primary site, liver-limited metastases, disease status, RAS status. $P$-values $<0.050$ are shown in bold.

Abbreviations: CR, complete response; ECOG, Eastern Cooperative Oncology Group; OS, overall survival; PD, progressive disease; PFS, progression-free survival; PR, partial response; PS, performance status; SD, stable disease; SNPs, single nucleotide polymorphisms. 


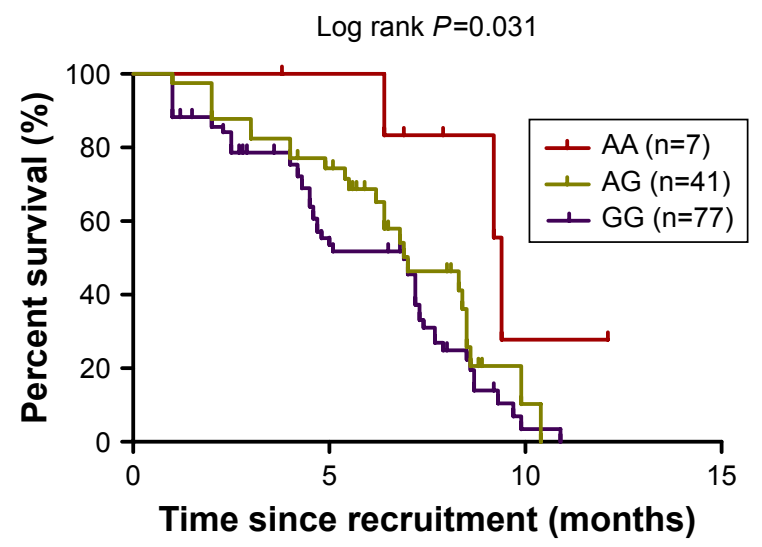

Figure I Kaplan-Meier curves of PFS according to the three variants (AA, AG, and GG) of MLHI rsI800734 SNPs in mCRC patients treated by first-line irinotecanbased chemotherapy.

Abbreviations: mCRC, metastatic colorectal cancer; PFS, progression-free survival; SNPs, single nucleotide polymorphisms.

Furthermore, the PFS of AA + AG combined variants and GG variant of MLH1 rs1800734 SNPs was 8.3 months and 6.9 months (log-rank $P=0.037$ ). In univariate analysis, the result showed statistically significant difference between two groups (HR: 1.61; 95 CI: 1.02-2.55; $P=0.043$, Figure 3), whereas no statistically significant difference was found in multivariable analysis (HR: 1.57; 95 CI: 0.98-2.53; $P=0.061)$.

\section{Discussion}

To our knowledge, this is the first study to set forth the relationship between the different variants of MLH1 rs1800734 SNPs and clinical outcomes of the first-line irinotecanbased chemotherapy in mCRC patients. Our results showed that compared with GG variant, the AA variant of MLH1

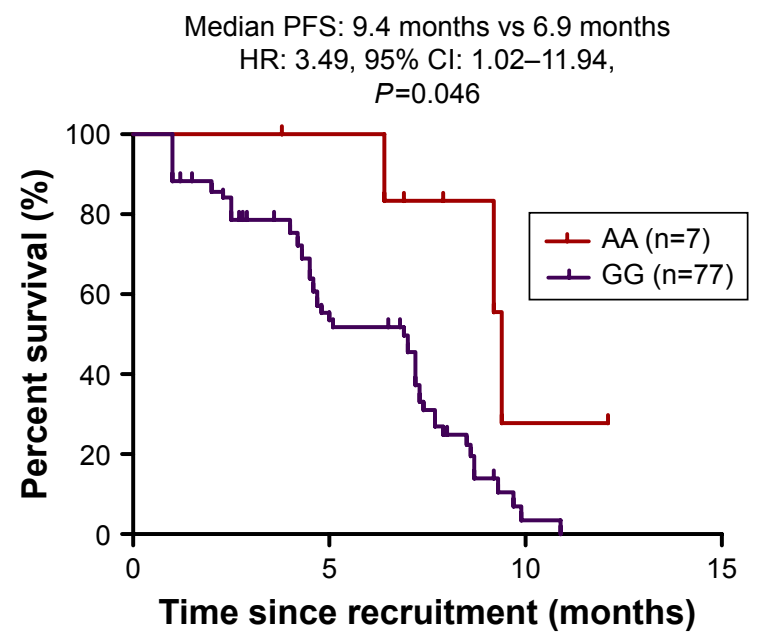

Figure 2 Kaplan-Meier curves of PFS according to AA variant compared with GG variant of MLHI rs 1800734 SNPs in $\mathrm{mCRC}$ patients treated by first-line irinotecanbased chemotherapy.

Abbreviations: $\mathrm{mCRC}$, metastatic colorectal cancer; PFS, progression-free survival; SNPs, single nucleotide polymorphisms.
Median PFS 8.3 months vs 6.9 months

HR: $1.57,95 \% \mathrm{Cl}: 0.98-2.53$ $P=0.061$

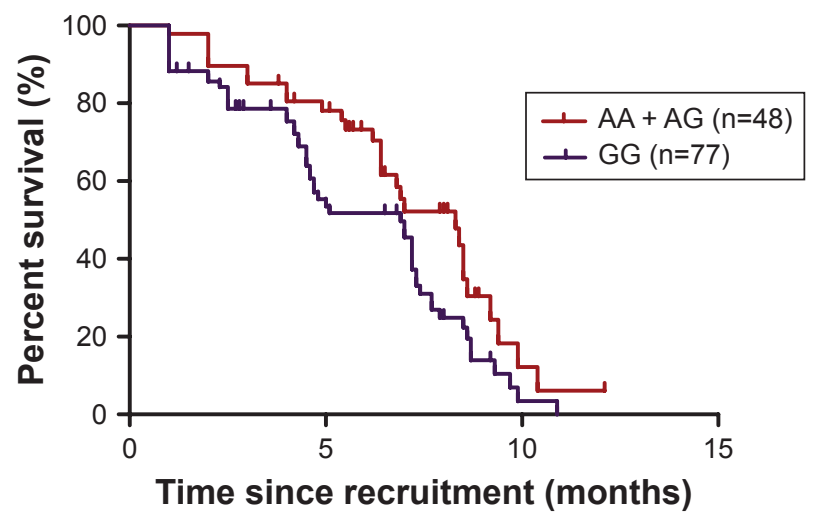

Figure 3 Kaplan-Meier curves of PFS according to AA + AG combined variants compared with GG variant of MLHI rs 1800734 SNPs in mCRC patients treated by first-line irinotecan-based chemotherapy.

Abbreviations: mCRC, metastatic colorectal cancer; PFS, progression-free survival; SNPs, single nucleotide polymorphisms.

rs 1800734 SNPs has a better PFS and no statistically significant in ORR analysis.

Nowadays, the gold standard of judgment for MMR status was confirmed by protein immunohistochemistry of MLH1, MSH2, MSH6, and PMS2.${ }^{17}$ Gene point mutation and large fragment deletion during DNA replication in human normal cells were repaired by proficient MMR system. ${ }^{9}$ As known, irinotecan was catalyzed by carboxylesterase and transformed into 7-ethyl-10-hydroxycamptothecin 38(SN-38) in body, interfering DNA replication and transcription to exert antitumor activity. ${ }^{18}$ We hypothesized that MMR system can involve in repair of the abnormal DNA replication caused by irinotecan-based chemotherapy, and the dMMR system could not play its normal function, leading to a better response to cytotoxic chemotherapy. This assumption had been brought up in previous studies, ${ }^{12,13}$ which was the same as the synthetic lethality theory from PARP inhibitors in Brcal mutation cancers. ${ }^{19,20}$

Due to extensive genome-wide associative studies, researchers found lots of genomic susceptibility loci, in which some of them played an important role in the development of CRC, including MLH1 rs1800734 SNPs in this article. ${ }^{21,22}$ Previous study found that A allele of MLH1 rs1800734 had a higher hypermethylation level in gene promoter of MLH1 than G allele, causing MLH1 protein expression decrease and changing the function of MMR system. ${ }^{12}$ This finding may partly explain why AA variant of MLH1 rs1800734 SNPs is associated with longer PFS and any A allele of MLH1 rs1800734 SNPs with higher ORR compared with GG variant, which is the result of our article. Some researches explored the relationship between SNPs of other genes and 
clinical benefit of irinotecan-based therapy, such as TOP 1 , SLCO1B1, ABCC2, and UGT1A1. 23,24 Although the function of these genes mentioned above mainly take part in drugmetabolizing pathway in vivo, mechanisms affecting the clinical efficacy of irinotecan-based therapy were different from MLH1 rs1800734 SNPs in our study.

There are also some limitations in this article. As a retrospective study, inherent limitations make the results to be further confirmed by prospective studies. Second, as the follow-up time is insufficient, we only observe short-term efficacy of the influence of MLH1 SNPs on first-line irinotecan-based chemotherapy and cannot affirm whether different variants of MLH1 rs1800734 SNPs are related to OS or not. Although DNA synthetic lethality assumptions may explain the results of this article, we need to design in vitro cytology experiment in order to discover the mechanism behind.

Anyway, our results showed the AA variant of MLH1 rs1800734 SNPs have a longer PFS, which suggest that MLH1 rs1800734 SNPs maybe a predictive factor in first-line irinotecan-based chemotherapy for patients with $\mathrm{mCRC}$.

\section{Acknowledgments}

The authors thank all patients who provided experiment samples generously. This work was funded by Foundation of Social Development Project of the Science and Technology Department of Jiangsu Province (BE2015719), Jiangsu Province social development key research and development plan (BE2017694), and Beijing Medical Award Foundation (YJHYXKYJJ-432).

\section{Disclosure}

The authors report no conflicts of interest in this work.

\section{References}

1. Chen W, Zheng R, Baade PD, et al. Cancer statistics in China, 2015. CA Cancer J Clin. 2016;66(2):115-132.

2. Douillard JY, Cunningham D, Roth $\mathrm{AD}$, et al. Irinotecan combined with fluorouracil compared with fluorouracil alone as first-line treatment for metastatic colorectal cancer: a multicentre randomised trial. Lancet. 2000;355(9209):1041-1047.

3. de Gramont A, Figer A, Seymour M, et al. Leucovorin and fluorouracil with or without oxaliplatin as first-line treatment in advanced colorectal cancer. J Clin Oncol. 2000;18(16):2938-2947.

4. Saltz LB, Cox JV, Blanke C, et al. Irinotecan plus fluorouracil and leucovorin for metastatic colorectal cancer. Irinotecan Study Group. $N$ Engl J Med. 2000;343(13):905-914.
5. Singh A, Singh N, Behera D, Sharma S. UGT1A1*6 polymorphisms are correlated with irinotecan-induced neutropenia: a systematic review and meta-analysis. Med Oncol. 2017;34(4):64.

6. Xu C, Tang X, Qu Y, et al. UGT1A1 gene polymorphism is associated with toxicity and clinical efficacy of irinotecan-based chemotherapy in patients with advanced colorectal cancer. Cancer Chemother Pharmacol. 2016;78(1):119-130.

7. Innocenti $\mathrm{F}$, Undevia SD, Iyer L, et al. Genetic variants in the UDPglucuronosyltransferase $1 \mathrm{~A} 1$ gene predict the risk of severe neutropenia of irinotecan. J Clin Oncol. 2004;22(8):1382-1388.

8. Vilar E, Gruber SB. Microsatellite instability in colorectal cancer-the stable evidence. Nat Rev Clin Oncol. 2010;7(3):153-162.

9. Sargent DJ, Marsoni S, Monges G, et al. Defective mismatch repair as a predictive marker for lack of efficacy of fluorouracil-based adjuvant therapy in colon cancer. J Clin Oncol. 2010;28(20):3219-3226.

10. Le DT, Uram JN, Wang H, et al. PD-1 blockade in tumors with mismatch-repair deficiency. $N$ Engl J Med. 2015;372(26):2509-2520.

11. Overman MJ, Mcdermott R, Leach JL, et al. Nivolumab in patients with metastatic DNA mismatch repair-deficient or microsatellite instabilityhigh colorectal cancer (CheckMate 142): an open-label, multicentre, phase 2 study. Lancet Oncol. 2017;18(9):1182-1191.

12. Ma J, Zhang Y, Shen H, et al. Association between mismatch repair gene and irinotecan-based chemotherapy in metastatic colon cancer. Tumour Biol. 2015;36(12):9599-9609.

13. Kim JE, Hong YS, Ryu MH, et al. Association between deficient mismatch repair system and efficacy to irinotecan-containing chemotherapy in metastatic colon cancer. Cancer Sci. 2011;102(9):1706-1711.

14. Ma G, Ge Y, Gu D, et al. Functional annotation of colorectal cancer susceptibility loci identifies MLH1 rs1800734 associated with MSI patients. Gut. 2016;65(7):1227-1228.

15. Trojan J, Zeuzem S, Randolph A, et al. Functional analysis of hMLH1 variants and HNPCC-related mutations using a human expression system. Gastroenterology. 2002;122(1):211-219.

16. Xiao XQ, Gong WD, Wang SZ, et al. Polymorphisms of mismatch repair gene hMLH1 and hMSH2 and risk of gastric cancer in a Chinese population. Oncol Lett. 2012;3(3):591-598.

17. Jiricny J. The multifaceted mismatch-repair system. Nat Rev Mol Cell Biol. 2006;7(5):335-346.

18. Fujita K, Kubota $Y$, Ishida H, Sasaki Y. Irinotecan, a key chemotherapeutic drug for metastatic colorectal cancer. World J Gastroenterol. 2015;21(43):12234-12248.

19. Srinivasan G, Sidhu GS, Williamson EA, et al. Synthetic lethality in malignant pleural mesothelioma with PARP1 inhibition. Cancer Chemother Pharmacol. 2017;80(4):861-867.

20. Heerma van Voss MR, Brilliant JD, Vesuna F, et al. Combination treatment using DDX3 and PARP inhibitors induces synthetic lethality in BRCA1-proficient breast cancer. Med Oncol. 2017;34(3):33.

21. Tanikawa C, Kamatani Y, Takahashi A, et al. GWAS identifies two novel colorectal cancer loci at 16q24.1 and 20q13.12. Carcinogenesis. 2018; 39(5):652-660.

22. Liu NQ, Ter Huurne M, Nguyen LN, et al. The non-coding variant rs1800734 enhances DCLK3 expression through long-range interaction and promotes colorectal cancer progression. Nat Commun. 2017;8: 14418.

23. Paolicchi E, Vivaldi C, de Gregorio V, et al. Topoisomerase 1 promoter variants and benefit from irinotecan in metastatic colorectal cancer patients. Oncology. 2016;91(5):283-288.

24. Teft WA, Welch S, Lenehan J, et al. OATP1B1 and tumour OATP1B3 modulate exposure, toxicity, and survival after irinotecan-based chemotherapy. Br J Cancer. 2015;112(5):857-865. 


\section{Publish your work in this journal}

OncoTargets and Therapy is an international, peer-reviewed, open access journal focusing on the pathological basis of all cancers, potential targets for therapy and treatment protocols employed to improve the management of cancer patients. The journal also focuses on the impact of management programs and new therapeutic agents and protocols on

patient perspectives such as quality of life, adherence and satisfaction. The manuscript management system is completely online and includes a very quick and fair peer-review system, which is all easy to use. Visit http://www.dovepress.com/testimonials.php to read real quotes from published authors.

Submit your manuscript here: http://www.dovepress.com/oncotargets-and-therapy-journal 